\title{
Evaluating the prognostic significance of preoperative thrombocytosis in epithelial ovarian cancer
}

\author{
S.K. Allensworth, MD ${ }^{1}$, C.L. Langstraat, MD ${ }^{1}$, J.R. Martin, RN ${ }^{2}$, M.A. Lemens, RN, BSN ${ }^{2}$, \\ M.E. McGree, BS ${ }^{3}$, A.L. Weaver, MS $^{3}$, S.C. Dowdy, MD $^{1}$, K.C. Podratz, MD, PhD ${ }^{1}$, and J.N. \\ Bakkum-Gamez, MD1 \\ ${ }^{1}$ Department of Obstetrics and Gynecology, Division of Gynecologic Surgery Mayo Clinic, \\ Rochester, Minnesota \\ ${ }^{2}$ Department of Surgical Clinical Research, Mayo Clinic, Rochester, Minnesota \\ ${ }^{3}$ Division of Biomedical Statistics and Informatics, Mayo Clinic, Rochester, Minnesota
}

\begin{abstract}
Objective-Preoperative thrombocytosis has been implicated as a negative prognostic marker for epithelial ovarian cancer (EOC). We assessed whether thrombocytosis is an independent risk factor for EOC recurrence and death.

Methods-Perioperative patient characteristics and process-of-care variables (National Surgical Quality Improvement Program (NSQIP)-defined) were retrospectively abstracted from 587 women who underwent EOC staging between 1/2/03-12/29/08. Thrombocytosis was defined as platelet count $>450 \times 10^{9} / \mathrm{L}$. Disease-free survival (DFS) and overall survival (OS) were determined using Kaplan-Meier methods. Associations were evaluated with Cox proportional hazards regression and hazard ratios (HR).
\end{abstract}

Results-The incidence of preoperative thrombocytosis was 22.3\%. DFS was $70.8 \%$ and $36.0 \%$ at 1 and 3 years. OS was $83.3 \%$ and $54.3 \%$ at 1 and 3 years. Ascites, lower hemoglobin, advanced disease, and receipt of perioperative packed red blood cell transfusion were independently associated with thrombocytosis. Older age and the presence of coronary artery disease were associated with lower likelihood of thrombocytosis. Overall, thrombocytosis was an independent predictor of increased risk of recurrence. Among early stage (I/II) cases, there was a 5-fold increase in the risk of death and nearly 8 -fold risk of disease recurrence independently associated with thrombocytosis.

Conclusion-Preoperative thrombocytosis portends worse DFS in EOC. In early stage disease, thrombocytosis is a potent predictor of worse DFS and OS and further assessment of the impact of circulating platelet-derived factors on EOC survival is warranted. Thrombocytosis is also associated with extensive initial disease burden, measurable residual disease, and postoperative sequelae. Preoperative platelet levels may have value in primary cytoreduction counseling.

(C) 2013 Elsevier Inc. All rights reserved.

Address Correspondence to: Jamie Bakkum-Gamez, M.D., Eisenberg Lobby 71, Mayo Clinic, 200 First Street, SW, Rochester, MN 55905, 507-538-5867 (phone), 507-266-9300 (fax), Bakkum.jamie@ mayo.edu.

The authors declare that there are no conflicts of interest.

Publisher's Disclaimer: This is a PDF file of an unedited manuscript that has been accepted for publication. As a service to our customers we are providing this early version of the manuscript. The manuscript will undergo copyediting, typesetting, and review of the resulting proof before it is published in its final citable form. Please note that during the production process errors may be discovered which could affect the content, and all legal disclaimers that apply to the journal pertain. 


\section{Introduction}

Approximately 95\% of ovarian cancers are of epithelial origin and in 2012, there were 22,280 estimated new diagnoses of ovarian cancer and 15,500 deaths from the disease [1]. Serologic measurements including cancer antigen 125 (CA-125), albumin, hemoglobin, and platelet levels, have emerged as potential preoperative predictors of outcome [2-7]. The rate of preoperative thrombocytosis in primary epithelial ovarian cancer (EOC) has been reported to range from $31-42 \%[2,3]$. Thrombocytosis has been linked to poor prognosis in patients with esophageal and colorectal carcinoma suggesting that platelets may have a direct role in tumor progression [8, 9]. Additionally, "paraneoplastic thrombocytosis" has recently been implicated in playing a causal role in poor prognosis EOC $[2,10,11]$.

Platelets have been shown to be associated with cancer progression, invasion, and metastasis $[5,8,12-14]$. Once activated, platelets may impact cancer evolution via mechanisms that offer tumor cell protection and potentiate tumor and vascular growth [12, 13, 15]. Some of these mechanisms can enhance disease progression and ultimately affect prognosis. Platelets produce or augment growth factors and enzymes, such as lysophosphatidic acid, matrix metalloproteinases, thymidine phosphorylase, and vascular endothelial growth factors (VEGF) which play a role in angiogenesis, tumor cell extravasation, and tumor cell invasion and metastasis $[4,8,12,13,15]$. Recently, serum thrombopoietin and interleukin-6 (IL-6) levels were shown to be elevated in patients with advanced stage EOC and thrombocytosis and platelets from those women with EOC-associated thrombocytosis abrogated the effect of taxane chemotherapy in vitro [2]. In addition, in mouse EOC models, silencing both thrombopoietin and IL- 6 expression resulted in resolution of thrombocytosis, and neutralizing IL-6 made EOC more sensitive to taxane therapy [2]. Mechanistically, TGF $\beta$ R 1 and COX-1 also appear to play a role in EOC paraneoplastic thrombocytosis [10]. However, thrombocytosis has consistently been shown to be associated with more advanced stage and higher grade cancers, and shorter overall survival may alternatively be directly related to disease burden and biology [2, 3].

It remains unclear as to whether prognosis is affected directly by the presence of a high level of platelets or if thrombocytosis is a surrogate for other prognostic indicators, such as higher disease burden, higher residual disease (RD), or even perioperative complications. Prior studies evaluating thrombocytosis in EOC have presented limited cohort sizes and/or clinical data, making it difficult to control for other factors that may impact thrombocytosis or independently influence prognosis. In addition, the impact of preoperative thrombocytosis on surgical complexity and perioperative management in EOC patients has not been described. In this study, we explored whether preoperative thrombocytosis is independently associated with EOC prognosis and whether aggressive surgical debulking negates the association between preoperative thrombocytosis and poorer prognosis.

\section{Methods}

Perioperative data was collected retrospectively on women who underwent primary cytoreduction for EOC, primary peritoneal cancer (PPC), or fallopian tube cancer (FTC) between 1/2/03 and 12/29/08 at the Mayo Clinic in Rochester, Minnesota. All cases of EOC, PPC and FTC were considered under the umbrella of EOC for this study. Patients were excluded if they underwent neoadjuvant therapy, were being treated for recurrent disease, underwent prior surgery for their cancer, or had non-epithelial or non-ovarian malignancy. This study was approved by the Institutional Review Board of Mayo Foundation. In accordance with the Minnesota Statute for Use of Medical Information in Research, women who did not consent to the use of their medical records for research purposes were also excluded. 
Perioperative National Surgical Quality Improvement Program (NSQIP) defined variables that could potentially influence platelet count and disease outcomes were identified and data abstracted by a trained, dedicated registered nurse abstractor. Variables included, but were not limited to, patient characteristics such as age, body mass index (BMI), Eastern Cooperative Oncology Group (ECOG) performance status, American Society of Anesthesiologists (ASA) score, smoking history, medical comorbidities, preoperative laboratory data (hemoglobin, platelets, creatinine, albumin, CA-125), presence of ascites, initial disease extent, operative complexity (previously defined) [16], extent of RD after primary cytoreduction, estimated blood loss (EBL) and perioperative packed red blood cell transfusion (PRBCT) status, tumor histology, surgical stage and grade, postoperative complications during initial hospitalization including venous thromboembolism (VTE), cardiac events, or central nervous system (CNS) events.

Preoperative thrombocytosis was defined as a platelet count greater than $450 \times 10^{9} / \mathrm{L}$ prior to surgery [2]. The primary outcomes were disease-free survival (DFS) and overall survival (OS). Both outcomes were analyzed among all patients regardless of debulking status.

The statistical analysis was performed using the SAS software package Version 9.2 (SAS Institute, Inc.; Cary, NC). Patient characteristics, process-of-care variables, disease-specific parameters, and postoperative complications were summarized using standard descriptive statistics. Each variable was evaluated separately in a logistic regression model for its association preoperative thrombocytosis. A parsimonious multivariable model was identified using stepwise and backward variable selection considering the patient characteristics, process-of-care variables, and disease-specific variables with a p-value $<0.20$ based on univariate analysis. Variables with a p-value $<0.05$ were retained in the final multivariable model. Associations were summarized by calculating odds ratios (OR) and corresponding 95\% confidence intervals (CI) using the parameter estimates and standard errors from the logistic regression models. For DFS, duration of follow-up was calculated from the date of surgery to the date of recurrence or last follow-up. For OS, duration of follow-up was calculated from the date of surgery to the date of death or last follow-up. DFS and OS were estimated using the Kaplan-Meier method. Each factor was evaluated separately in a Cox proportional hazards regression model for its association with DFS and OS, respectively. Variables with a p-value $<0.20$ univariately were allowed to compete in multivariable modeling, with the exception of preoperative thrombocytosis. A multivariable Cox model was determined using stepwise and backward variable selection methods. Variables with a $\mathrm{p}$-value $<0.05$ were retained in the final model. As the final step, the variable for preoperative thrombocytosis was included in each final model in order to assess its prognostic value over and above the other variables. Associations with DFS and OS were summarized by calculating hazard ratios (HR) and corresponding 95\% confidence intervals (CI) using the parameter estimates and standard errors from the Cox models. Adjusted survival curves were constructed to compare the adjusted survival for patients with, versus without, preoperative thrombocytosis using the partially model-free approach described by Storer et al [25]. The estimates were adjusted for the factors included in each final multivariable model.

\section{Results}

\section{Patient characteristics and demographics}

There were 587 women that underwent primary EOC staging and debulking between 1/2/03 and 12/29/08 and consented to their medical records being used for research purposes. Nine women were excluded as they did not have a documented preoperative platelet count. Based on the 578 women eligible for the study, the mean platelet count was $376.9 \times 10^{9} / \mathrm{L}$ (SD, $146.3)$ and $129(22.3 \%)$ had preoperative thrombocytosis. Stage distribution was as follows: 
$90(15.6 \%)$ stage I, $37(6.4 \%)$ stage II, 347 (60.0\%) stage III, $104(18.0 \%)$ stage IV. At the time of surgery, $312(54.0 \%)$ were debulked to microscopic RD and 181 (31.3\%) were debulked to $\leq \mathrm{cm}$ but measurable disease. Thus, $493(85.3 \%)$ received an optimal cytoreduction by contemporary definition [17]. Among those optimally debulked, 127 $(25.8 \%)$ were stage I/II and $366(74.2 \%)$ were stage III/IV. Among the stage I/II cohort, $117 / 127(92.1 \%)$ underwent comprehensive surgical staging, including bilateral salpingooophorectomy, hysterectomy (if uterus present), bilateral pelvic and para-aortic lymphadenectomy, and omentectomy. Among stage I/II patients the mean platelet count was $318.3 \times 10^{9} / \mathrm{L}(\mathrm{SD}, 109.5)$ with $6.3 \%$ (8/127) having thrombocytosis and among stage III/IV patients the mean platelet count was $393.4 \times 10^{9} / \mathrm{L}(\mathrm{SD}, 151.1)$ with $26.8 \%$ (121/451) having thrombocytosis. Detailed cohort characteristics and demographics have been previously published [20].

In the overall cohort of 578 women, a total of 314 deaths have been documented (stage I/II: 5 and 18 , respectively, among the 8 with and 119 without thrombocytosis; stage III/IV: 90 and 201, respectively, among the 121 with and 330 without thrombocytosis). The median time to death for the 314 women was 1.98 (interquartile range (IQR) $0.80,3.30$ ) years. Among those alive at last follow-up, the median follow-up was 2.95 (IQR $0.73,4.89$ ) years. A total of 296 women have had a documented recurrence (stage I/II: 5 and 20, respectively, among those with and without thrombocytosis; stage III/IV: 88 and 183, respectively, among those with and without thrombocytosis). The median time to recurrence among these 296 women was 1.11 (IQR 0.71, 1.63) years. Among those who have not recurred, median follow-up was 2.180 .44 (IQR 0.1207, 4.233.27) years.

\section{Perioperative factors associated with preoperative thrombocytosis}

In univariate analysis, preoperative thrombocytosis was associated with ASA score 23 , lower preoperative hemoglobin, lower albumin, advanced stage disease, high grade, and serous histology. Thrombocytosis was associated with the presence of ascites and more extensive disease involvement, including omentum, diaphragm, splenic, small and large bowel mesentery, bowel serosa, as well as bladder peritoneum disease. Those with thrombocytosis were more likely to have undergone a debulking with high operative complexity, yet were also more likely to have measureable RD at the end of their cytoreduction. Thrombocytosis was also associated with higher EBL, receipt of PRBCT, and longer operating time (Table S1). Table 1 summarizes the patient characteristics, process-ofcare variables, and disease-specific variables that were identified as significantly associated with preoperative thrombocytosis in the multivariable analysis.

Preoperative thrombocytosis was also associated with adverse sequelae, with higher observed rates of postoperative ileus (34.9\% vs $21.6 \%$ in those without thrombocytosis, $\mathrm{p}=0.002)$, surgical site infection $(10.9 \%$ vs $3.6 \%, \mathrm{p}=0.001)$, and urinary tract infection $(11.6 \%$ vs $6.0 \%, \mathrm{p}=0.03)$. In fact, the risk of any postoperative complication during the initial hospitalization was higher $(48.8 \%$ vs $32.1 \%, \mathrm{p}<0.001)$ and the length of initial hospitalization was longer $(\mathrm{p}<0.001)$ in those with thrombocytosis (Table $\mathrm{S} 1$ ).

\section{Thrombocytosis and risk of recurrence}

Within the entire cohort, preoperative thrombocytosis was associated with increased risk of recurrence (HR 2.37 [95\% CI 1.82, 3.09]) on univariate analyses (Table S2; Figure 1a). Additionally, age, greater ASA score, higher preoperative CA-125, the presence of ascites, residual disease, increasing EBL, receipt of PRBCT, serous histology, advanced stage, higher grade, and higher surgical complexity were also associated with increased risk of recurrence on univariate analyses. Development of a postoperative mechanical bowel obstruction or leak, ileus, or surgical site infection was also associated with increasing risk 
of recurrence. Higher preoperative hemoglobin was associated with a decreased risk of recurrence (Table S2). In a multivariable analysis, preoperative thrombocytosis was also an independent predictor of worse DFS within the overall cohort, as were the presence of ascites, residual disease, and advanced stage (Table 2). Adjusted time-to-event analyses demonstrated worse DFS among those with thrombocytosis (Figure 1a).

Additionally, when we stratified the cohort into early stage (I/II) and advanced stage (III/IV) disease, preoperative thrombocytosis was independently associated with worse DFS in early stage disease with a nearly 8-fold increase in risk of recurrence (HR 7.90 [95\% CI 2.49, 25.06]) (Table 2; Figure 1b). In advanced stage disease, thrombocytosis was not an independent predictor of increased recurrence after adjusting for the other factors, (HR 1.31 [95\% CI 0.99, 1.75) (Table 2; Figure 1c).

\section{Thrombocytosis and overall survival}

Thrombocytosis was associated with an increased risk of death (HR 1.76 [95\% CI 1.38, 2.24]) in univariate analysis (Table S3; Figure 2a). Additionally, increasing age and worse ECOG performance status, ASA score $\geq 3$, a past medical history of hypertension, chronic obstructive pulmonary disease (COPD)/emphysema, higher preoperative creatinine and CA-125, the presence of ascites, measurable RD, higher EBL, receipt of a PRBCT, serous histology, advanced stage, and higher grade were also associated with decreased OS. Postoperative sequelae that were associated with an increased risk of death included development of a surgical site infection, urinary tract infection, deep vein thrombosis/ pulmonary embolism (DVT/PE), or the occurrence of a cardiac event. Higher preoperative albumin and higher preoperative hemoglobin, as well as intermediate surgical complexity, were associated with an improved OS (Table S3).

We performed multivariate analyses utilizing the variables presented by Stone, et al. [2] as well as a separate analysis in which all variables with a $p<0.20$ in univariate analysis were allowed to compete (Table 3$)$. When allowing all variables $(p<0.20)$ to compete, regardless of stage, thrombocytosis was no longer significantly associated with a decrease in OS. Increasing age, ECOG performance status $\geq 2$, ASA score $\geq 3$, the presence of ascites, measurable RD, advanced stage, and development of DVT/PE were independently associated with an increased risk of death. Serous histology was associated with improved OS (Table 3). When we limited our multivariate OS analysis to the variables included by Stone, et al. [2], we found thrombocytosis to be significantly associated with death (adjusted HR 1.45 [95\% CI 1.13, 1.85]). Also, using the variables published by Stone, et al. [2], increasing age, advanced stage ( 지IA), higher grade, and RD $>1 \mathrm{~cm}$ were significantly associated with poorer OS (Table 3).

When we stratified the cohort into early stage (I/II) and advanced stage (III/IV), multivariate OS analysis revealed a different set of independent risk factors for each strata. In early stage disease, preoperative thrombocytosis (adjusted HR 5.06 [95\% CI 1.31, 14.73] emerged as a potent independent OS prognostic indicator (Figure 2b) as did the occurrence of a postoperative surgical site infection or cardiac event (Table 4).

Among those with advanced stage disease, factors influencing OS continued to be those of overall health and conditioning (age, ECOG performance status, ASA score), postoperative DVT/PE, presence of ascites, and RD (Table 4). Adjusted time-to-event analyses in the entire cohort (Figure 2a) and advanced stage disease (Figure 2c) revealed no difference in OS between those with and without thrombocytosis. 


\section{Discussion}

Preoperative thrombocytosis in women undergoing debulking and staging for EOC is wellrecognized and occurs in approximately one-third of all cases [2, 3, 5, 18-21]. The prognostic significance of thrombocytosis in the setting of a new EOC diagnosis, however, has not been clear. The relationship between platelet function and outcomes in EOC appears to be complex. Having elevated platelets at the time of EOC diagnosis is associated with well-established poor prognostic markers such as advanced stage, measurable RD, and postoperative complications [22-24] and, when controlling for such variables, outcomes in advanced stage disease appear to be marginally influenced by preoperative thrombocytosis. However, in the setting of early stage disease, thrombocytosis appears to be a powerful prognostic marker for both recurrence and death with a risk of recurrence of almost 8-fold in early stage cases with thrombocytosis compared to those without thrombocytosis. This suggests that systemic factors exist that are not counteracted by cytoreduction and are resistant to chemotherapy. Among the 127 stage I/II patients there were only 8 with preoperative thrombocytosis, therefore the confidence limits for the hazard ratio estimates are rather wide and findings should be cautiously interpreted.

Platelets have been implicated by others as a culprit in chemoresistance. Recent evidence has shown that incubating platelets with ovarian cancer cell lines increases cell proliferation [10] and decreases chemotherapeutic efficacy [11]. Additionally, infusing platelets into orthotopic EOC mouse models enhances tumor growth [10]. There appears to be a link between serum thrombopoietin and IL-6 and thrombocytosis in women with EOC; additionally, modulating both thrombopoietin and IL-6 levels in a mouse EOC model enhanced taxane efficacy [2]. TGF $\beta R 1$ also appears to have a critical role, and COX-1 may also contribute to the effect of paraneoplastic thrombocytosis [10]. Taken together, these findings suggest a causal relationship between thrombocytosis and disease response to cytotoxic chemotherapy making novel agents that target the active effects of platelets intriguing.

Among those with advanced stage disease, thrombocytosis does not seem to be as potent of a predictor for DFS, and does not independently impact OS. Perhaps this is due to an overwhelming influence of other clinical factors, such as extent of disease and presence of $\mathrm{RD}$ in those with advanced stage disease. Others have demonstrated that thrombocytosis is associated with advanced stage disease and higher CA-125 levels, both of which are welldocumented to be associated with worse prognosis [2, 3, 5, 18, 19]. However, we also found that thrombocytosis was associated with poorer preoperative health (ASA score), more extensive disease requiring more complex surgical efforts, and a higher likelihood of postoperative adverse events, such as ileus, surgical site infection, and urinary tract infection. One could postulate that, while the presence of abnormally elevated platelets does not independently impact OS, platelets and their inherent growth factors $[4,8,12,13,15]$ have a systemic effect on overall health, cancer growth, gastrointestinal recovery, and/or immune responses to surgical stress. Alternately, poorer preoperative health and/or extensive cancer may trigger an abnormal rise in platelet levels and may be the causative factor(s) in postoperative sequelae. While inherent patient and disease characteristics are not modifiable, reduction of postoperative complications may be possible through better understanding of cause and effect relationships. Additionally, given the relationship between preoperative thrombocytosis and postoperative sequelae, whether direct or indirect, platelet counts could be incorporated into preoperative patient counseling.

Strengths of this current study include the cohort size, which allowed us to control for multiple potential confounding variables. Patients underwent aggressive surgical management with over $85 \%$ undergoing cytoreduction down to $\leq \mathrm{cm}$ of RD and $54.0 \%$ of 
the entire cohort reaching microscopic RD. Additionally, over $92 \%$ of early stage cases underwent comprehensive staging, limiting the number of potentially understaged patients. When comparing clinical demographics between our cohort and the cohort from Stone, et al. there were some differences: thrombocytosis was present in $22.3 \%$ in our study and $31 \%$ of the reference cohort; stage III/IV disease made up $78.0 \%$ of our cohort and $91.6 \%$ of the reference cohort; we had $88.9 \%$ high grade cases while the reference cohort had $96.6 \%$ high grade; and, as noted above, the optimal cytoreduction rate for this current study was $85.3 \%$, compared to $57.9 \%$ for the reference cohort. [2]. Despite these differences, when we analyzed our cohort in concordance with the variables published by Stone et al., we obtained similar OS findings. Limitations of this study include the fact that it is a retrospective study with the usual limitations of dependence on accurate charting and documentation, lack of blinding, and potential for bias. In addition, this study is based on clinical outcomes and we did not explore potential mechanisms of cancer promotion in the platelets of our patients.

In conclusion, preoperative thrombocytosis appears to be a potent poor prognostic indicator among women with EOC, especially in early stage disease. Emerging translational data suggests paraneoplastic thrombocytosis is causal in reduced survival. Further evaluation of circulating platelet-derived, cellular, and protein factors in EOC is warranted and platelettargeted therapeutic strategies should be explored. In advanced stage disease, thrombocytosis does not appear to impact OS and preoperative platelet levels should not influence the surgical approach to resectable disease. However, preoperative thrombocytosis may be predictive of initial disease burden, residual disease, and adverse postoperative sequelae. Further characterization of these relationships could guide counseling regarding neoadjuvant chemotherapy and/or yield modification strategies to reduce short-term morbidity.

\section{Supplementary Material}

Refer to Web version on PubMed Central for supplementary material.

\section{Acknowledgments}

This work was supported in part by the Office of Women's Health Research Building Interdisciplinary Careers in Women's Health (BIRCWH award K12 HD065987).

\section{References}

1. Siegel R, ND, Jemal A. Cancer statistics. CA Cancer J Clin. 2012; 62(1):10-29. [PubMed: 22237781]

2. Stone RL, et al. Paraneoplastic Thrombocytosis in Ovarian Cancer. New England J of Medicine. 2012; 366(7):610-618.

3. Gungor T, K-PM, Sucak A, Mollamahmutoglu L. The role of thrombocytosis in prognostic evaluation of epithelial ovarian tumors. Arch Gynecol Obstet. 2009; 279(1):53-56. [PubMed: 18470520]

4. Wheler J, TAM, Hong D, Naing A, Jackson T, Liu S, Feng L, Kurzrock R. Survival of patients in a Phase 1 clinic. Cancer. 2009; 115:1091-1099. [PubMed: 19165805]

5. de Jong D, EMJ, Lie Fong S, Gerestein CG, Kooi GS, Baalbergen A, van der Burg MEL, Burger $\mathrm{CW}$, Ansink AC. Preoperative predictors for residual tumor after surgery in patients with ovarian carcinoma. Oncology. 2007; 72(5-6):293-301. [PubMed: 18198490]

6. Richardson DL, MA, Cliby WA. Risk factors for anastomotic leak after recto-sigmoid resection for ovarian cancer. Gynecol Oncol. 2006; 103(2):667-672. [PubMed: 16797684]

7. Herzog T, et al. Correlation between CA-125 serum level and response to RECIST in a phase III ovarian cancer study. Gynecol Oncol. 2011; 122(2):350-355. [PubMed: 21546066] 
8. Aminian A, KF, Mirsharifi R, Alibakhshi A, Dashti H, Jahangiri Y, Safari S, Ghaderi H, Noaparast M, Hasani SM, Mirsharifi A. Significance of platelet count in esophageal carcinomas. Saudi J Gastroenterol. 2011; 17(2):134-137. [PubMed: 21372352]

9. Sasaki K, KK, Tsuno NH, Sunami E, Kitayama J. mpact of preoperative thrombocytosis on the survival of patients with primary colorectal cancer. World J Surg. 2012; 36(1):192-200. [PubMed: 22045447]

10. Cho MS, et al. Platelets increase the proliferation of ovarian cancer cells. Blood. 2012

11. Radziwon-Balicka A, et al. Platelets increase survival of adenocarcinoma cells challenged with anticancer drugs: mechanisms and implications for chemoresistance. British Journal of Pharmacology. 2012; 167(4):787-804. [PubMed: 22506717]

12. Gupta GP, Massague J. Platelets and metastasis revisited: a novel fatty link. J Clin Invest. 2004; 114(12):1691-1693. [PubMed: 15599391]

13. Holmes CE, LJE, Ornstein DL. Activated platelets enhance ovarian cancer cell invasion in cellular model of metastasis. Clin Exp Metastasis. 2009; 26(7):653-661. [PubMed: 19444623]

14. Li AJ, KBY. Androgen mediation of thrombocytosis in epithelial ovarian cancer biology. Clin Cancer Res. 2005; 11(22):8015-8018. [PubMed: 16299230]

15. Manenti L, PP, Floriani I, Landoni F, Torri V, Buda A, Taraboletti G, Labianca R, Belotti D, Giavazzi R. Expression levels of vascular endothelial growth factor, matrix metalloproteinases 2 and 9 and tissue inhibitor of metalloproteinases 1 and 2 in the plasma of patients with ovarian carcinoma. Eur J Cancer. 2003; 39(13):1948-1956. [PubMed: 12932675]

16. Aletti GD, et al. Relationship among surgical complexity, short-term morbidity, and overall survival in primary surgery for advanced ovarian cancer. American Journal of Obstetrics and Gynecology. 2007; 197(6):676.e1-676.e7. [PubMed: 18060979]

17. Khoury-Collado FCDS. Recent surgical management of ovarian cancer. J Obstet Gynaecol Res. 2011; 37(5):379-82. [PubMed: 21463433]

18. Li AJ, MAC, Cass IC, Leuchter RS, Lagasse LD, Karlan BY. The prognostic significance of thrombocytosis in epithelial ovarian carcinoma. Gynecol Oncol. 2004; 92(1):211-214. [PubMed: 14751160]

19. Lee M, et al. The impact of pretretament thrombocytosis and persistent thrombocytosis after adjuvant chemotherapy in patients with advanced epithelial ovarian cancer. Gynecol Oncol. 2011; 122(2):238-241. [PubMed: 21531448]

20. Zeimet AG, MC, Muller-Holzner E, Daxenbichler G, Dapunt O. Significance of thrombocytosis in patients with epithelial ovarian cancer. AJOG. 1994; 170(2):549-554.

21. Crasta JA, PTS, Krishnan SM, Vallikad E, Rameshkumar K. Significance of preoperative thrombocytosis in epithelial ovarian cancer. Indian J Pathol Microbiol. 2010; 53(1):54-56. [PubMed: 20090223]

22. Aletti GD, et al. Aggressive Surgical Effort and Improved Survival in Advanced-Stage Ovarian Cancer. Obstet Gynecol. 2006; 107(1):77-85. [PubMed: 16394043]

23. Elattar A, et al. Optimal primary surgical treatment for advanced epithelial ovarian cancer. Cochrane Database Syst Rev. 2011; (8):CD007565. [PubMed: 21833960]

24. Ozols RF, et al. Phase III Trial of Carboplatin and Paclitaxel Compared With Cisplatin and Paclitaxel in Patients With Optimally Resected Stage III Ovarian Cancer: A Gynecologic Oncology Group Study. Journal of Clinical Oncology. 2003; 21(17):3194-3200. [PubMed: 12860964]

25. Storer BE, Gooley TA, Jones MP. Adjusted Estimates for Time-to-Event Endpoints. LIfetime Data Anal. 2008; 14(4):484-495. [PubMed: 18791867] 


\section{Highlights}

- Preoperative thrombocytosis portends worse disease-free survival in ovarian cancer.

- Patients with early stage disease and preoperative thrombocytosis have a higher risk of recurrence and death.

- Preoperative thrombocytosis is associated with higher initial disease burden, residual disease, and adverse postoperative sequelae. 

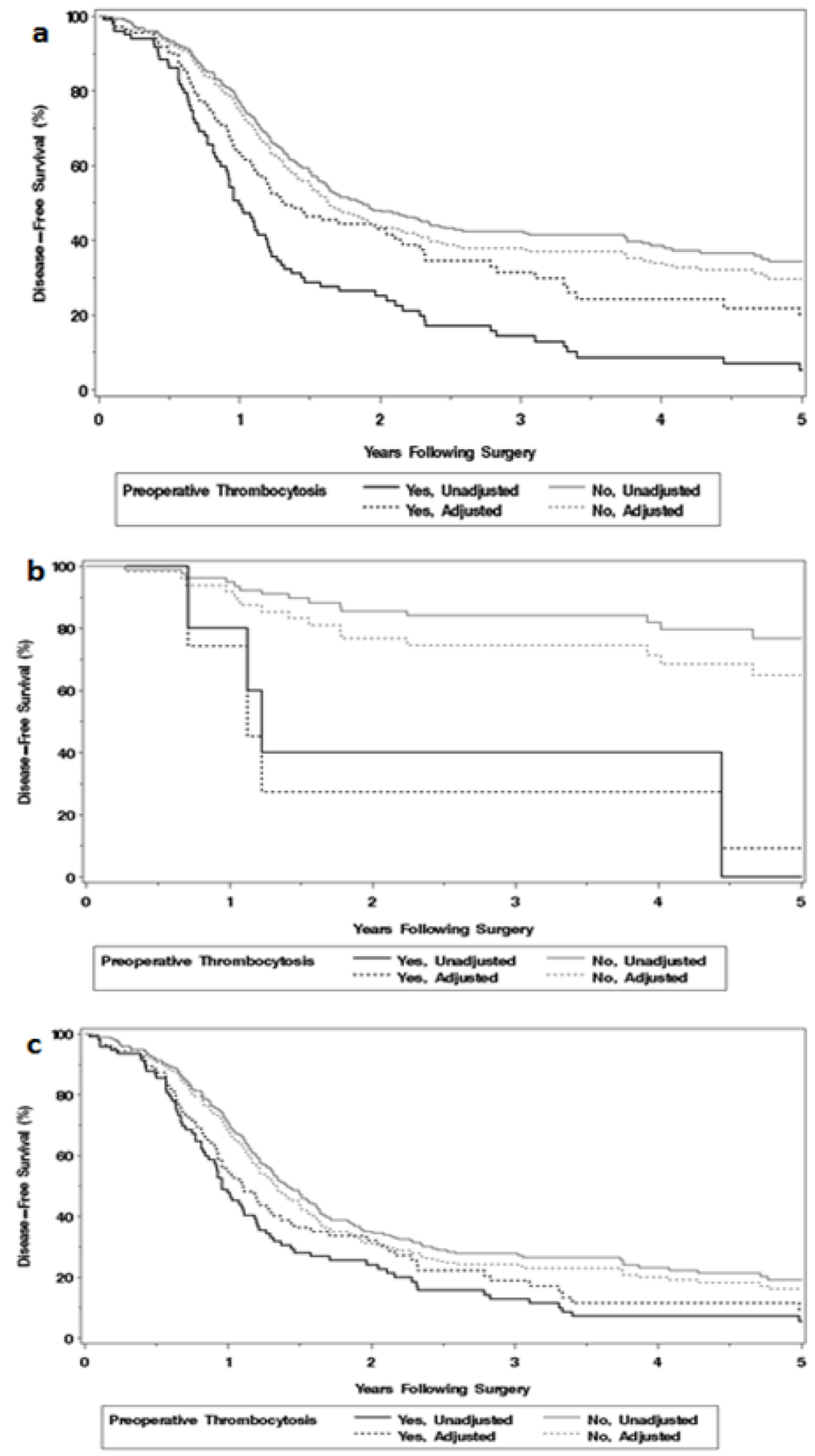

Figure 1.

Adjusted and unadjusted disease-free survival according to preoperative thrombocytosis in a) entire cohort $(\mathrm{N}=578)$, b) early stage (I/II) disease $(\mathrm{N}=127)$, and c) advanced stage (III/ IV) disease $(\mathrm{N}=451)$. 

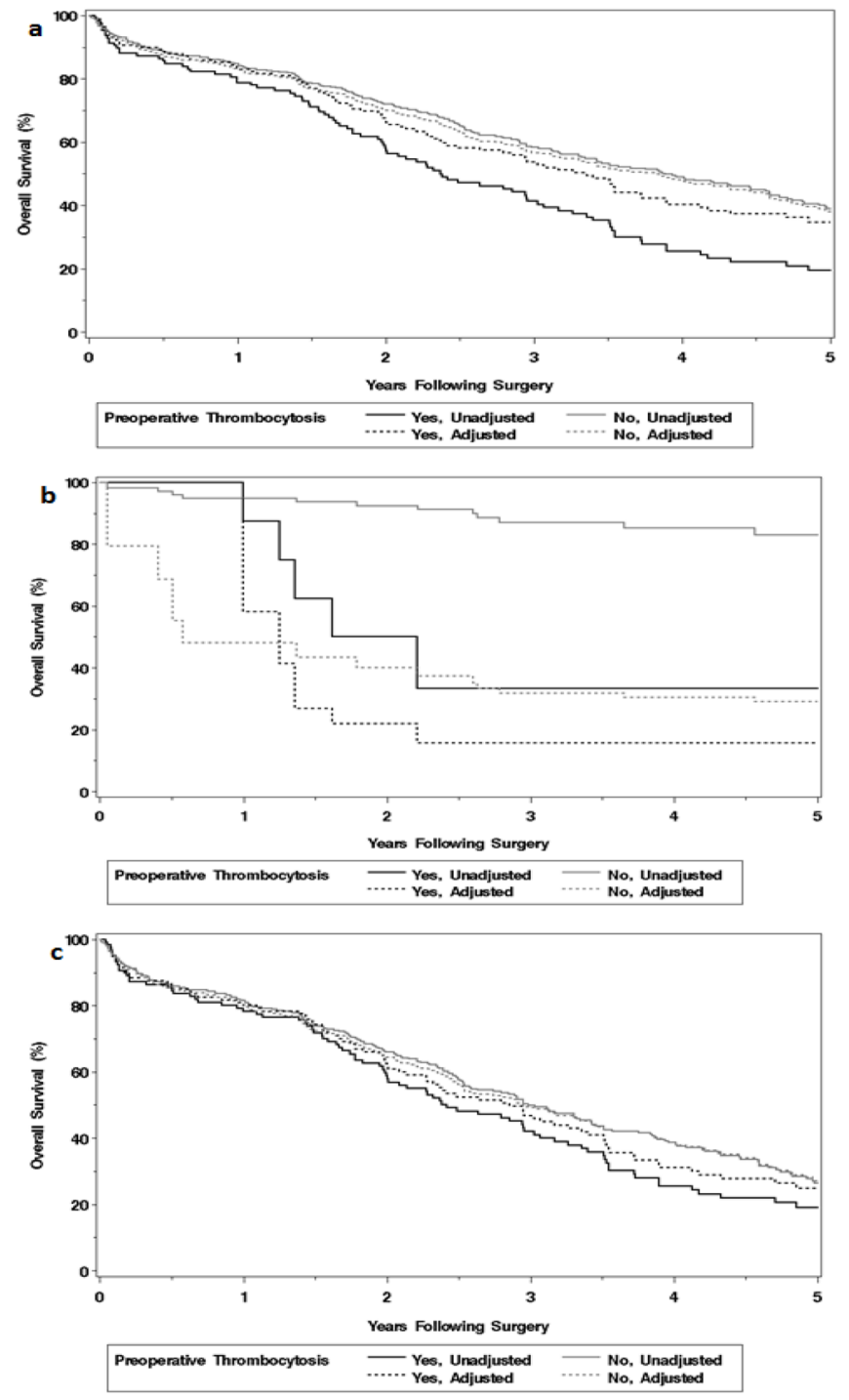

Figure 2.

Adjusted and unadjusted overall survival according to preoperative thrombocytosis in a) entire cohort ( $\mathrm{N}=578)$, b) early stage (I/II) disease ( $\mathrm{N}=127)$, and c) advanced stage (III/IV) disease $(\mathrm{N}=451)$. 


\section{Table 1}

Multivariable analysis of perioperative factors associated with preoperative thrombocytosis.

\begin{tabular}{lcc}
\hline Characteristic & Adjusted OR (95\% CI) & P value \\
\hline Age (years) ${ }^{\dagger}$ & $0.78(0.64,0.94)$ & 0.01 \\
Coronary artery disease & $0.24(0.08,0.73)$ & 0.01 \\
Preoperative hemoglobin $(\mathrm{g} / \mathrm{dL})^{\dagger}$ & $0.71(0.61,0.84)$ & $<0.001$ \\
Ascites & & $<0.001$ \\
$\quad$ No & Reference & \\
Yes & $3.62(2.10,6.24)$ & \\
Packed red blood cell transfusion & & \\
$\quad$ No & Reference & \\
Yes & $2.64(1.19,5.86)$ & \\
FIGO stage & \\
I/II & Reference \\
$\quad>$ IIIA & $3.23(1.45,7.23)$ \\
\hline & \\
\hline &
\end{tabular}


Table 2

Multivariable analysis of factors associated with disease-free survival, stratified by stage.

\begin{tabular}{|c|c|c|}
\hline Characteristic & Adjusted HR (95\% CI) & P value \\
\hline \multicolumn{3}{|l|}{ All stages $(\mathrm{N}=578)$} \\
\hline Ascites & $1.78(1.32,2.41)$ & $<0.001$ \\
\hline Residual disease & & $<0.001$ \\
\hline None & Reference & \\
\hline Yes, measurable ( $\triangleleft \mathrm{cm})$ & $1.53(1.14,2.05)$ & \\
\hline Yes, suboptimal or extensive $(>1 \mathrm{~cm})$ & $2.10(1.44,3.05)$ & \\
\hline FIGO stage & & $<0.001$ \\
\hline $\mathrm{I} / \mathrm{II}$ & Reference & \\
\hline પ્રIIA & $3.37(2.07,5.47)$ & \\
\hline Preoperative platelets & & 0.02 \\
\hline$\leq 450 \times 10^{9} / \mathrm{L}$ & Reference & \\
\hline$>450 \times 10^{9} / \mathrm{L}$ & $1.38(1.04,1.82)$ & \\
\hline \multicolumn{3}{|l|}{ Stage I/II (N=127) } \\
\hline Ascites & $3.95(1.55,10.05)$ & 0.004 \\
\hline Preoperative platelets & & $<0.001$ \\
\hline$\leq 450 \times 10^{9} / \mathrm{L}$ & Reference & \\
\hline$>450 \times 10^{9} / \mathrm{L}$ & $7.90(2.49,25.06)$ & \\
\hline \multicolumn{3}{|l|}{$\underline{\text { Stage III/IV }(N=451)}$} \\
\hline Ascites & $1.693(1.24,2.31)$ & $<0.001$ \\
\hline Residual disease & & $<0.001$ \\
\hline None & Reference & \\
\hline Yes, measurable ( $\triangleleft \mathrm{cm})$ & $1.56(1.16,2.09)$ & \\
\hline Yes, suboptimal or extensive $(>1 \mathrm{~cm})$ & $2.15(1.48,3.13)$ & \\
\hline Preoperative platelets & & 0.06 \\
\hline$\leq 450 \times 10^{9} / \mathrm{L}$ & Reference & \\
\hline$>450 \times 10^{9} / \mathrm{L}$ & $1.31(0.99,1.75)$ & \\
\hline
\end{tabular}




\section{Table 3}

Multivariable analysis of factors associated with overall survival. The analysis model was built based on variables that were significant in univariate analysis in this study. The reference model demonstrates the multivariable overall survival analysis in our cohort using the variables from Stone, et al. [2].

\begin{tabular}{|c|c|c|c|c|}
\hline \multirow{2}{*}{ Characteristic } & \multicolumn{2}{|l|}{ Analysis model } & \multicolumn{2}{|l|}{ Reference model } \\
\hline & Adjusted HR (95\% CI) & $P$ value & Adjusted HR (95\% CI) & P value \\
\hline Age (years) ${ }^{*}$ & $1.27(1.14,1.41)$ & $<0.001$ & $1.33(1.20,1.47)$ & $<0.001$ \\
\hline ECOG performance status & & $<0.001$ & & \\
\hline 0 & Reference & & - & \\
\hline 1 & $1.23(0.92,1.65)$ & & - & \\
\hline $2+$ & $2.98(2.07,4.29)$ & & - & \\
\hline ASA score & & 0.005 & & \\
\hline 1 or 2 & Reference & & - & \\
\hline $3+$ & $1.44(1.12,1.85)$ & & - & \\
\hline Postoperative DVT/PE & $2.14(1.32,3.46)$ & 0.002 & - & \\
\hline Ascites & $2.14(1.61,2.85)$ & $<0.001$ & - & \\
\hline Residual disease & & $<0.001$ & & $<0.001$ \\
\hline None & Reference & & Reference & \\
\hline Yes, measurable ( $\triangleleft \mathrm{cm})$ & $1.17(0.88,1.56)$ & & Reference & \\
\hline Yes, suboptimal or extensive $(>1 \mathrm{~cm})$ & $2.13(1.54,2.95)$ & & $2.16(1.65,2.83)$ & \\
\hline Histology & & 0.006 & & 0.09 \\
\hline Non-serous & Reference & & Reference & \\
\hline Serous & $0.65(0.49,0.88)$ & & $0.78(0.58,1.04)$ & \\
\hline FIGO stage & & $<0.001$ & & $<0.001$ \\
\hline $\mathrm{I} / \mathrm{II}$ & Reference & & Reference & \\
\hline ل्राIIA & $2.80(1.73,4.53)$ & & $3.25(2.05,5.16)$ & \\
\hline Preoperative platelets & & 0.22 & & 0.003 \\
\hline$\leq 450 \times 10^{9} / \mathrm{L}$ & Reference & & Reference & \\
\hline$>450 \times 10^{9} / \mathrm{L}$ & $1.18(0.91,1.52)$ & & $1.45(1.13,1.85)$ & \\
\hline FIGO grade & & & & 0.03 \\
\hline 1 or 2 & - & & Reference & \\
\hline 3 & - & & $2.00(1.09,3.68)$ & \\
\hline
\end{tabular}


Table 4

Multivariable analysis of factors associated with overall survival, stratified by stage.

\begin{tabular}{|c|c|c|}
\hline Characteristic & Adjusted HR (95\% CI) & $P$ value \\
\hline \multicolumn{3}{|l|}{ Stage I/II $(\mathrm{N}=127)^{* *}$} \\
\hline Preoperative platelets & & 0.008 \\
\hline$\leq 450 \times 10^{9} / \mathrm{L}$ & Reference & \\
\hline$>450 \times 10^{9} / \mathrm{L}$ & $5.06(1.31,14.73)$ & \\
\hline Postoperative surgical site infection & $9.07(1.29,61.84)$ & 0.03 \\
\hline Postoperative cardiac event & $218.18(25.89,2702.26)$ & $<0.001$ \\
\hline \multicolumn{3}{|l|}{ Stage III/IV (N=451) } \\
\hline Age (years) ${ }^{*}$ & $1.26(1.12,1.40)$ & $<0.001$ \\
\hline ECOG performance status & & $<0.001$ \\
\hline 0 & Reference & \\
\hline 1 & $1.16(0.86,1.58)$ & \\
\hline $2+$ & $2.92(2.01,4.24)$ & \\
\hline ASA score & & 0.006 \\
\hline 1 or 2 & Reference & \\
\hline $3+$ & $1.44(1.11,1.87)$ & \\
\hline Ascites & $1.84(1.38,2.46)$ & $<0.001$ \\
\hline Residual disease & & $<0.001$ \\
\hline None & Reference & \\
\hline Yes, measurable $(\triangleleft \mathrm{cm})$ & $1.15(0.87,1.54)$ & \\
\hline Yes, suboptimal or extensive $(>1 \mathrm{~cm})$ & $2.12(1.53,2.93)$ & \\
\hline Preoperative platelets & & 0.29 \\
\hline$\$ 450 \times 10^{9} / \mathrm{L}$ & Reference & \\
\hline$>450 \times 10^{9} / \mathrm{L}$ & $1.15(0.89,1.50)$ & \\
\hline Postoperative DVT/PE & $2.27(1.40,3.67)$ & $<0.001$ \\
\hline Per 10-year increase in age & & \\
\hline
\end{tabular}

